\title{
Estudo da doença sarcopênica em indivíduos adultos com sobrepeso e obesidade
}

\author{
Study of sarcopenic disease in overweight and obese adult individuals \\ Estudio de la enfermedad sarcopénica en adultos con sobrepeso y obesidad
}

Recebido: 01/12/2021 | Revisado: 08/12/2021 | Aceito: 10/12/2021 | Publicado: 17/12/2021

\author{
Cleison Bonfim Dias \\ ORCID: https://orcid.org/0000-0002-1361-4082 \\ Faculdade Independente do Nordeste, Brasil \\ E-mail: cleisson.bd113@gmail.com \\ Virgílio Santana Júnior \\ ORCID: https://orcid.org/0000-0003-1062-2584 \\ Faculdade Independente do Nordeste, Brasil \\ E-mail: virgiliofisio@hotmail.com
}

\begin{abstract}
Resumo
Este trabalho visa trazer dados sobre o estudo da doença sarcopênica, sobre a obesidade e sobre a obesidade sarcopênica, tendo como objetivo avaliar a presença da sarcopenia em indivíduos adultos com sobrepeso ou obesidade. Trata-se de um estudo transversal e analítico de abordagem quantitativa em 100 indivíduos adultos (50 obesos e 50 sobrepesos), entre 18 e 60 anos de idade. Os estudos foram realizados em uma clínica particular na cidade de Vitória da Conquista - BA. Contudo, ao findar das aplicações dos questionários e coletar os dados dos indivíduos, esses foram tabulados e receberam tratamento estatístico descritivo e analítico. A amostra apresentou predominância do sexo feminino tanto em sobrepesos quanto para obesos, não havendo diferença estatística significante entre os grupos para o sexo. Mesmo com as limitações da pesquisa, foi possível atingir os objetivos e trazer resultados concretos que indivíduos jovens com padrão de sobrepeso ou obesidade podem e apresentam sarcopenia. Ainda sugere-se a realização de novos estudos afim de abordar mais evidências científicas em indivíduos obesos e sobrepesos com sarcopenia.
\end{abstract}

Palavras-chave: Sarcopenia; Obesidade; Obesidade sarcopênica.

\begin{abstract}
This work aims to bring data on the study of sarcopenic disease, obesity and sarcopenic obesity. This study aims to assess the presence of sarcopenia in overweight or obese adult individuals. This is a cross-sectional analytical study with a quantitative approach in 100 adult individuals (50 obese and 50 overweight), between 18 and 60 years of age. The studies were carried out in a private clinic in the city of Vitória da Conquista - BA. However, at the end of the application of the questionnaires and collecting data from the individuals, they were tabulated and received descriptive and analytical statistical treatment. The sample showed a predominance of females both in overweight and obese, with no statistically significant difference between groups for gender. Even with the limitations of the research, it was possible to achieve the objectives and bring concrete results that young individuals with a pattern of overweight or obesity can and do have sarcopenia. Further studies are suggested in order to address more scientific evidence in obese and overweight individuals with sarcopenia.
\end{abstract}

Keywords: Sarcopenia; Obesity; Sarcopenic obesity.

\section{Resumen}

Este trabajo tiene como objetivo aportar datos sobre el estudio de la enfermedad sarcopénica, la obesidad y la obesidad sarcopénica. Este estudio tiene como objetivo evaluar la presencia de sarcopenia en adultos con sobrepeso u obesidad. Se trata de un estudio analítico transversal con abordaje cuantitativo en 100 individuos adultos (50 obesos y 50 con sobrepeso), entre 18 y 60 años. Los estudios se realizaron en una clínica privada de la ciudad de Vitória da Conquista - BA. Sin embargo, al finalizar la aplicación de los cuestionarios y la recolección de datos de los individuos, se tabularon y recibieron tratamiento estadístico descriptivo y analítico. La muestra mostró un predominio del sexo femenino tanto en sobrepeso como en obesidad, sin diferencia estadísticamente significativa entre grupos por género. Incluso con las limitaciones de la investigación, fue posible alcanzar los objetivos y obtener resultados concretos de que los jóvenes con un patrón de sobrepeso u obesidad pueden y tienen sarcopenia. Se sugieren más estudios para abordar más evidencia científica en personas obesas y con sobrepeso con sarcopenia.

Palabras clave: Sarcopenia; Obesidad; Obesidad sarcopénica. 


\section{Introdução}

A sarcopenia é a perda da massa muscular esquelética, associada à redução da força muscular ou desempenho físico. Ela acomete indivíduos ao longo dos anos e é potencializada por fatores como doenças ou estilo de vida inadequado. Entre as consequências, está o aumento da ocorrência de quedas e limitação para atividades da vida diária- (Rocha, 2018).

Estudos epidemiológicos sugerem que diferentes fatores contribuem para o desenvolvimento da sarcopenia, incluindo alterações hormonais, perda de neurônios motores, nutrição inadequada, inatividade física e baixo grau de inflamação crônica. Essas alterações têm sido apresentadas até mesmo em indivíduos saudáveis, fisicamente ativos, resultando em perda da massa muscular de, aproximadamente, 1\% a 2\% por ano, a partir dos 50 anos de idade- (Dreyer, 2005 apud Pícoli et al., 2015).

A obesidade pode ser definida, de forma simplificada, como doença caracterizada pelo acúmulo excessivo de gordura corporal, sendo consequência do balanço energético positivo e que acarreta repercussões à saúde, com perda importante na qualidade e no tempo de vida- (Tavares et al., 2010).

Campos et al., 2018) nos fala que a obesidade relacionada a sarcopenia tem um grande impacto na qualidade de vida dos idosos e de qualquer indivíduo que sofre com essa comorbidade, pois acarreta em redução nas atividades de vida diária, na independência funcional e gera incapacidade.

Contudo, vimos que, de fato, a associação da obesidade com a sarcopenia causa vários fatores relevantes para uma boa qualidade de vida e que quando os indivíduos são acometidos com tais comorbidades a sobrevida é ainda menor, já que alguns autores citados acima nos mostram que o nível de decadência dos pacientes é tão exacerbado que provoca até o óbito. Assim o estudo visa através de ferramentas de avaliação identificar a existência ou não da sarcopenia nos participantes adultos e jovens.

Frente ao exposto, este estudo apresenta como objetivo avaliar a presença da sarcopenia em indivíduos adultos com sobrepeso ou obesidade.

\section{Metodologia}

Trata-se de um estudo transversal e analítico de abordagem quantitativa. Segundo Carvalho \& Rocha (2016), nos estudos transversais, cada indivíduo é avaliado para o fator de exposição e a doença em determinado momento. Muitas vezes o estudo transversal é realizado apenas com objetivo descritivo sem nenhuma hipótese para ser avaliada. Alguns têm usado o termo levantamento para denominar estudos transversais realizados com essa finalidade. O estudo transversal pode ser usado como um estudo analítico, ou seja, para avaliar hipóteses de associações entre exposição ou características e evento. No entanto limitações existem quando se tenta concluir qual a natureza da relação entre exposição e evento nestas situações. Essa limitação relaciona-se, principalmente, com o fato de que a exposição e a doença são avaliadas ao mesmo tempo (transversalmente). Ainda de acordo com os autores, uma questão importante que pode ficar sem resposta é sobre o que apareceu primeiro, o fator de exposição ou a doença. Um outro ponto que deve ser observado nestes estudos transversais é a dificuldade em separar os casos novos da doença dos casos já presentes por algum tempo. Desta forma esses estudos irão refletir não apenas determinantes de doença, mas, também, determinantes de sobrevida. Apesar das limitações, os estudos transversais, pelo fato de incluir indivíduos com e sem o evento e de poder avaliar associações entre o evento e exposições ou características, podem ser considerados um passo adiante na identificação dos determinantes de doenças, quando comparados com relatos ou séries de casos (Carvalho \& Rocha, 2016).

O estudo foi conduzido em uma clínica particular da cidade de Vitória da Conquista - Bahia, Brasil e foram incluídos na pesquisa adultos de ambos os sexos, com idade $\geq 18$ anos e $\leq 60$ anos de idade que aceitaram assinar o Termo de Consentimento Livre e Esclarecido (TCLE). 
A coleta de dados foi realizada através da aplicação das ferramentas de avaliação e um mini questionário. Os questionários foram aplicados em pacientes apresentando padrão de sobrepeso ou obesidade e as respostas foram depositadas no aparelho celular do próprio avaliador. Ainda realizaram um exame de bioimpedância e um teste de força muscular que estarão melhor descritos abaixo.

Todos os indivíduos selecionados nesse estudo foram submetidos a avaliações antropométricas. O índice de massa corpórea (IMC) foi calculado a partir da divisão do valor do peso corpóreo $(\mathrm{Kg})$ pela altura $(\mathrm{m})$ elevada ao quadrado. Os dados para cálculo do IMC foram obtidos com a utilização de uma balança digital com estadiômetro, calibrada, com resolução de 0,1 Kg para unidades de massa e de 0,1 cm para unidades de altura (Filizola®, Brasil). Os indivíduos foram posicionados sobre a balança com a cabeça orientada pelo plano de Frankfurt, em pé, descalços, formando um ângulo reto com estadiômetro. $\mathrm{O}$ indivíduo se posicionou com o corpo em posição ereta e calcanhares unidos.

Os critérios diagnósticos para a Sobrepeso e Obesidade foram baseados na avaliação do IMC dos indivíduos selecionados nesse estudo, a saber: peso normal (IMC entre 18 a 24,9 kg/m2), Sobrepeso (IMC entre 25 a $29,9 \mathrm{~kg} / \mathrm{m} 2$ ), Obesidade (IMC maior do que 30 kg/m2 sendo: Grau I: de 30 a 34,9 kg/m2; Grau II: de 35 a 39,9 kg/m2, Grau III: IMC maior do que $40 \mathrm{~kg} / \mathrm{m} 2$ ou $>35 \mathrm{~kg} / \mathrm{m} 2$ na presença de comorbidades) (OMS, 2004 apud Barbosa Filho et al., 2010).

Outras mensurações da composição corpórea investigadas foram:

- avaliação da circunferência da panturrilha esquerda (CP);

- avaliação da força muscular em flexão e extensão de quadril (FM);

- avaliação da velocidade da marcha (VM); e

- teste de bioimpedância elétrica (BE).

$\mathrm{Na}$ avaliação da bioimpedância elétrica (BE), esse é um método sensível de avaliação da composição corporal a partir de dados gerados pela resistência elétrica passiva do corpo a uma corrente elétrica de baixa amplitude $(500$ a $800 \mu \mathrm{A})$ e de alta frequência $(50 \mathrm{kHz})$, ajustados de acordo com dados de peso e estatura do indivíduo. A bioimpedância foi analisada de forma vetorial, utilizando-se o software BIVA. Ao final da medição com o aparelho, esse forneceu dados referentes ao percentual de gordura, massa gorda, massa magra, IMC e total e percentual de água corporal intra e extracelular, taxa metabólica basal e gasto diário de energia. Inicialmente, os indivíduos tiveram sua altura e peso aferidos no momento do exame. O indivíduo foi posicionado deitado em decúbito dorsal, com a colocação de quatro eletrodos em pontos anatômicos bem definidos e uniformemente posicionados. A colocação dos eletrodos foi próxima à articulação metacarpofalângea e entre os processos estiloides do rádio e da ulna da superfície dorsal da mão direita, e próximo à articulação metatarso-falangeana e entre os maléolos medial e lateral no dorso do pé direito.

De acordo com os dados obtidos com o percentual de massa gorda, os indivíduos foram classificados, de acordo com o sexo, em:

Homens: percentual de gordura normal (5 - 14,9); sobrepeso (15 - 19,9); obesidade moderada (20 - 24,9); obesidade grave (25 - 29,9); obesidade mórbida (> 29,9).

Mulheres: percentual de gordura normal (10-24,9); sobrepeso (25 - 29,9), obesidade moderada (30 - 34,9), obesidade grave $(35$ - 39,9), obesidade mórbida (> 39,9). 
As mensurações antropométricas e da composição corporal foram devidamente ajustadas de acordo com parâmetros idade e sexo. Cada parâmetro foi mensurado três vezes e o valor registrado correspondeu à média dos valores individuais coletados.

Velocidade de marcha: Indivíduos com Obesidade com capacidade de deambulação, com ou sem auxílio de anteparos, tiveram sua marcha medida em uma distância de 4 metros e acrescentando 1 metro imediatamente anterior e outro posterior aos 4 metros, eliminando os efeitos da aceleração e desaceleração. Receberam instruções para caminhar em velocidade superior a habitual, de maneira apressada, como se estivessem atravessando uma rua, sem, contudo, correrem. Cada indivíduo fez um total de três tentativas, sendo a velocidade máxima calculada pela média dessas medidas.

Avaliação do perímetro da panturrilha: Aqueles com valores de perimetria inferiores a $31 \mathrm{~cm}$ foram considerados sarcopênicos. Valores superiores ao estabelecido para FPM e/ ou perimetria de panturrilha caracterizaram os indivíduos com Obesidade como não sarcopênicos, já nos indivíduos com Obesidade que não tiverem condições físicas ou cognitivas para realizar o teste de velocidade de marcha, foi avaliada apenas a massa muscular (perimetria de panturrilha), considerando que para esse grupo de indivíduos com Obesidade, o resultado na velocidade de marcha seria $\leq 0,8 \mathrm{~m} / \mathrm{s}$.

Avaliação da Obesidade: Para definição da condição de obesidade entre os indivíduos selecionados, foi utilizado o Índice de Massa Corporal - IMC, obtido pela razão do peso corporal, em quilogramas, pela altura, em metros quadrados (peso/altura), considerando como ponto de corte para classificação do indivíduo com obesidade, valores de $\mathrm{IMC} \geq 30 \mathrm{Kg} / \mathrm{m}^{2}$ conforme recomendação proposta pela ("WHO | Obesity: preventing and managing the global epidemic", 2015) para classificar o estado nutricional de adultos e idosos.

O peso e a estatura foram avaliados com os indivíduos descalços, sem adornos na cabeça, utilizando roupas leves, posição ereta, pés juntos e braços posicionados ao longo do corpo, com a palma da mão voltada para a perna. Estas medidas foram avaliadas três vezes, sendo calculada a média entre os valores, caso houvesse diferença.

Para avaliação do peso foi utilizada balança de bioimpedância com variação de $0,1 \mathrm{~kg}$ e capacidade de até $150 \mathrm{~kg}$ e para a aferição da altura foi utilizado um estadiômetro portátil com capacidade: medição de $115 \mathrm{~cm}$ a $210 \mathrm{~cm}$; graduação: $1 \mathrm{~mm}$.

Avaliação da Força muscular: Os indivíduos deveriam estar em sedestação com quadris e joelhos fletidos a $90^{\circ} \mathrm{e}$ apoio das mãos na maca para evitar compensação da coluna, enquanto realizavam flexão de quadril com o aparelho localizado na região supra patelar e depois em decúbito ventral com joelhos flexionados também a $90^{\circ}$ com o aparelho na região posterior do fêmur distalmente realizando extensão do quadril. As medidas foram realizadas e repetidas por 3 vezes em cada lado, com pausas de 10 segundos entra essas, respeitando o tempo de descanso e evitando fadigas nos participantes.

Avaliação da Sarcopenia: Seguiram as diretrizes do Grupo de Trabalho Europeu sobre Sarcopenia (EWGSOP), que avalia três aspectos relacionados com a forma e função muscular esquelética dos indivíduos. A avaliação da sarcopenia foi realizada com base nos critérios do Consenso Europeu de Sarcopenia (Cruz-Jentoft et al, 2019) que classifica como: provável sarcopênico, indivíduos que apresentam diminuição de força muscular; sarcopênico, que apresentam diminuição de força muscular e de massa muscular; e, sarcopênicos severos, que apresentam diminuição de força muscular, massa muscular e performance física. No presente estudo optamos pela avaliação da força muscular; para massa muscular a composição corporal por Análise de Impedância Bioelétrica; e performance física pelo teste de velocidade de marcha.

A análise de Impedância Bioelétrica tetra polar se deu por meio de um aparelho portátil (RJL System Inc., modelo BIA101A, USA), que forneceu valores de resistência e reatância, com frequência de $50 \mathrm{kHz}$ e $800 \mu \mathrm{A}$.

A velocidade de marcha foi obtida pelo teste de velocidade de marcha de quatro metros, demarcados ao chão, no qual o indivíduo realizou o percurso dentro do seu padrão de marcha habitual e foi cronometrado o tempo de deslocamento. $\mathrm{O}$ teste de velocidade de marcha é validado e recomendado pelo Consenso Europeu de Sarcopenia, devido a conveniência de uso e 
capacidade de predizer resultados relacionados à sarcopenia. Foi realizado na área interna da clínica, em local que permita o indivíduo caminhar quatro metros em linha reta, superfície plana e sem obstáculos. Em nossa avaliação consideramos como uma velocidade baixa de marcha valores $\leq 0,8 \mathrm{~m} / \mathrm{s}$.

Os dados coletados foram tabulados e receberam tratamento estatístico descritivo (frequências, médias e medidas de dispersão) e analítico (Teste Qui-quadrado de Pearson e U de Mann- Whitney, ambos com significância fixa em 5\%) através do Statistical Package for the Social Sciences - SPSS, verão 22.2 para Windows. As tabelas e gráficos foram plotados pelo Microsoft Excel.

Este trabalho é um recorte do projeto com parecer número 2.653.020, respeitando os princípios éticos da resolução 466 de 12 de dezembro de 2012, item IV, do Conselho Nacional em Saúde.

\section{Resultados}

Para o estudo os 100 pacientes foram divididos igualmente entre os grupos obesos e sobrepesos. A amostra apresentou predominância do sexo feminino tanto em sobrepesos 35 (70,0\%), quanto para obesos 33 (66,0\%), não havendo diferença estatística significante entre os grupos para o sexo $(\mathrm{p}=0,415)$. Deste mesmo modo, não foi verificada diferença estatística significante para a idade $(\mathrm{p}=0,947)$, onde a faixa etária predominante para ambos os grupos esteve compreendida entre 54 e 56 anos, com $16(32,0 \%)$ dos indivíduos sobrepesos e 18 (36,0\%) para obesos, conforme esboça a Tabela 1.

Tabela 1. Características sociodemográficas e clínicas da amostra. Vitória da Conquista - BA, 2021.

\begin{tabular}{lccc}
\hline \multicolumn{1}{c}{ Variáveis } & \multicolumn{2}{c}{ Grupos, $n(\%)$} & $p^{*}$ \\
\hline Sexo & Sobrepesos $(\mathrm{n}=50)$ & Obesos $(\mathrm{n}=50)$ & \\
Masculino & $15(30,0)$ & $17(34,0)$ & 0,415 \\
Feminino & $35(70,0)$ & $33(66,0)$ & \\
Idade, anos & & & \\
$18-25$ & $4(8,0)$ & $2(4,0)$ & \\
$26-32$ & $6(12,0)$ & $7(14,0)$ & \\
$33-39$ & $9(18,0)$ & $8(14,0)$ & \\
$40-46$ & $8(16,0)$ & $8(16,0)$ & \\
$47-53$ & $7(14,0)$ & $18(36,0)$ & \\
$54-56$ & $16(32,0)$ &
\end{tabular}

*Teste do qui-quadrado de Pearson; Fonte: Dados da pesquisa.

Com auxílio do Gráfico 1, é possível inferir que o grupo obesidade apresentou para a perimetria da panturrilha média de $40,98( \pm 3,55) \mathrm{cm}$, sendo superior ao grupo sobrepreso com 37,62 $( \pm 2,90) \mathrm{cm}$. Desta forma a distribuição entre as médias foi verificada de forma significante $(\mathrm{p}<0,001)$. Por outro lado, os valores mínimos e máximos foram $32 \mathrm{~cm}$ e $49 \mathrm{~cm}$ para sobrepesos e 41,0 cm e 52,0 cm para obesos, não indicando condição satisfatória neste critério para ocorrência de sarcopenia entre os grupos. 
Gráfico 1. Distribuição da medida da perimetria da panturrilha pelo IMC da amostra. Vitória da Conquista - BA, 2021.

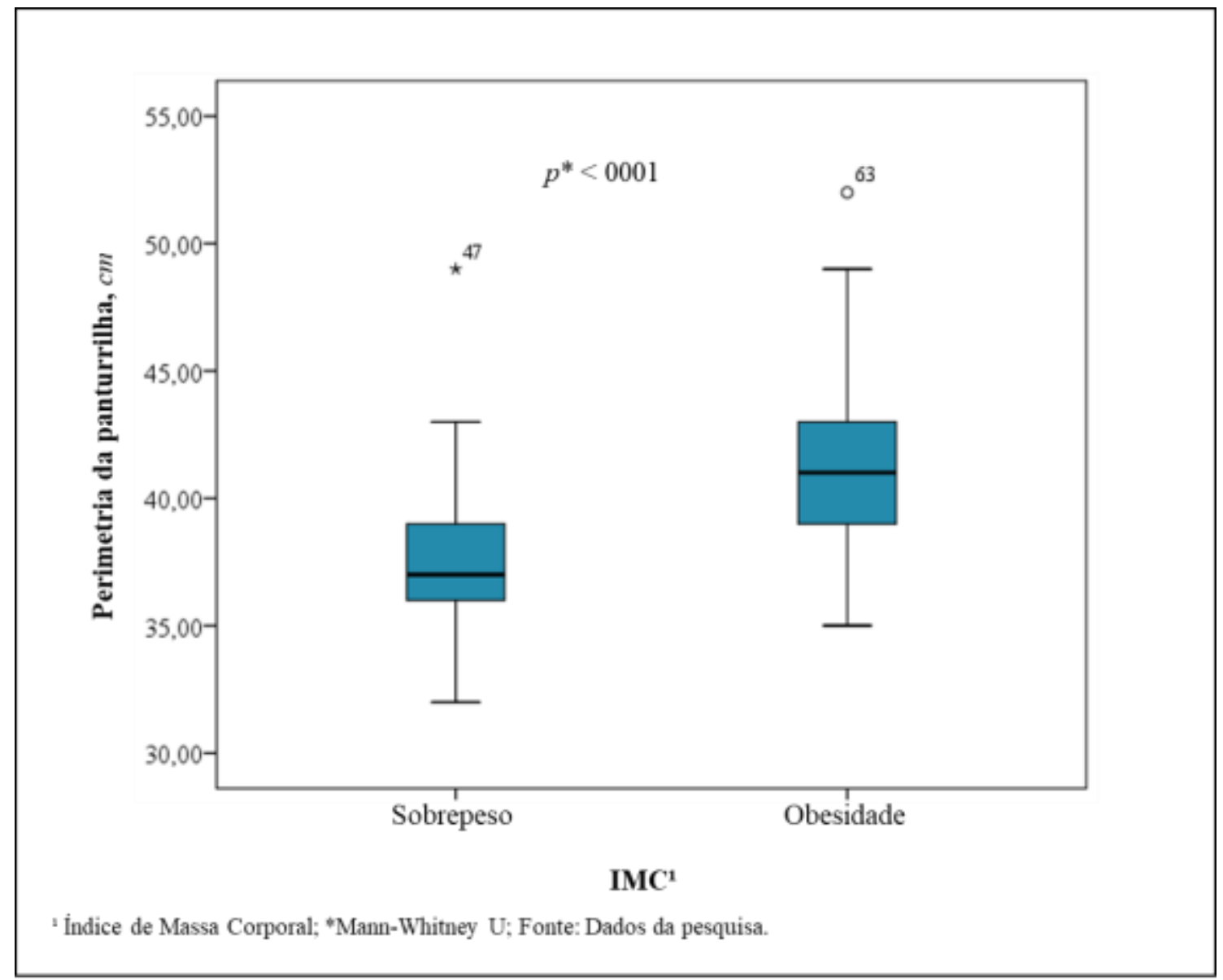

Fonte: Dados da pesquisa.

Ao analisar o Gráfico 2, é possível inferir que existe diferença significante entre a velocidade de marcha entre os grupos $(\mathrm{p}=0,04)$, onde sobrepesos apresentaram média de velocidade centrada em $0,60( \pm 0,10) \mathrm{m} / \mathrm{s}$, sendo superior à média dos obesos $0,57( \pm 0,78) \mathrm{m} / \mathrm{s}$. O grupo sobrepeso apresentou valor mínimo na velocidade da marcha de 0,34 m/s e máximo de $0,9 \mathrm{~m} / \mathrm{s}$, dessa forma a presença de outliers $(\mathrm{n}=2)$ e do limitante superior em $0,8 \mathrm{~m} / \mathrm{s}$, sugere a presença de 3 casos sem sarcopenia para os sobrepesos. Por outro lado, os obesos apresentaram valor mínimo de $0,37 \mathrm{~m} / \mathrm{s}$ e máximo de $0,78 \mathrm{~m} / \mathrm{s}$ sugerindo existência de sarcopenia em todo o grupo. 
Research, Society and Development, v. 10, n. 16, e503101624216, 2021

(CC BY 4.0) | ISSN 2525-3409 | DOI: http://dx.doi.org/10.33448/rsd-v10i16.24216

Gráfico 2. Distribuição da velocidade de marcha por IMC. Vitória da Conquista - BA, 2021.

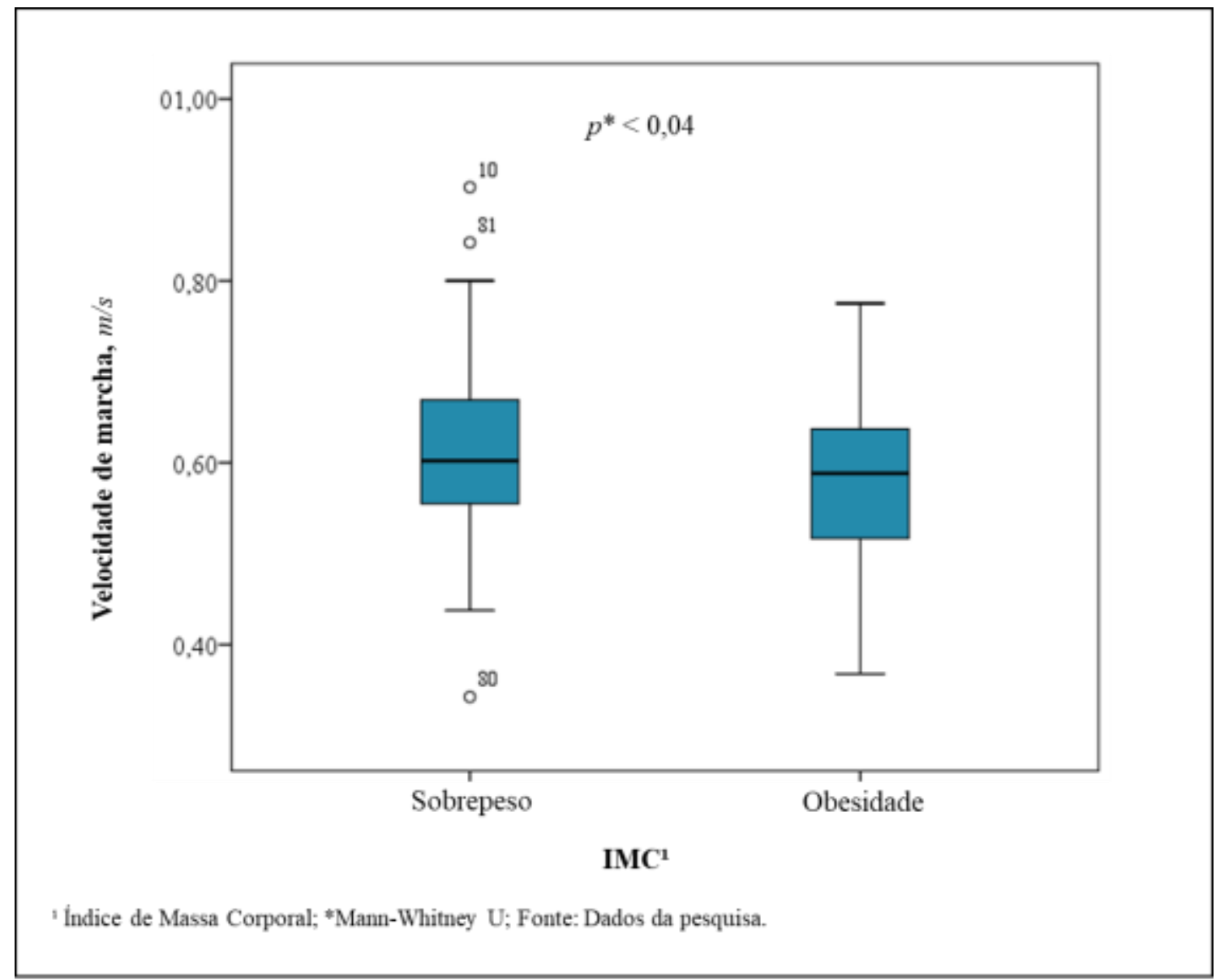

Fonte: Dados da pesquisa.

Com os dados contidos do Gráfico 3, infere-se que não foi verificada diferença estatística significante para a flexão entre sobrepesos e obesos tanto para lado esquerdo ( $\mathrm{p}=0,788)$, quanto para o lado direito $(0,341)$. Ressalta-se que para sobrepesos a média de força na flexão esquerdo foi de $17,04( \pm 5,15) \mathrm{kgf}$ e no direito de $17,04( \pm 5,40) \mathrm{kgf}$, sendo valores superiores aos dos obesos que apresentaram média de $14,99( \pm 4,90) \mathrm{kgf}$ e $15,97( \pm 5,11) \mathrm{kgf}$ para os lados esquerdo e direito, respectivamente. 
Gráfico 3. Distribuição da força dada pela flexão esquerda e direita por IMC. Vitória da Conquista - BA, 2021.

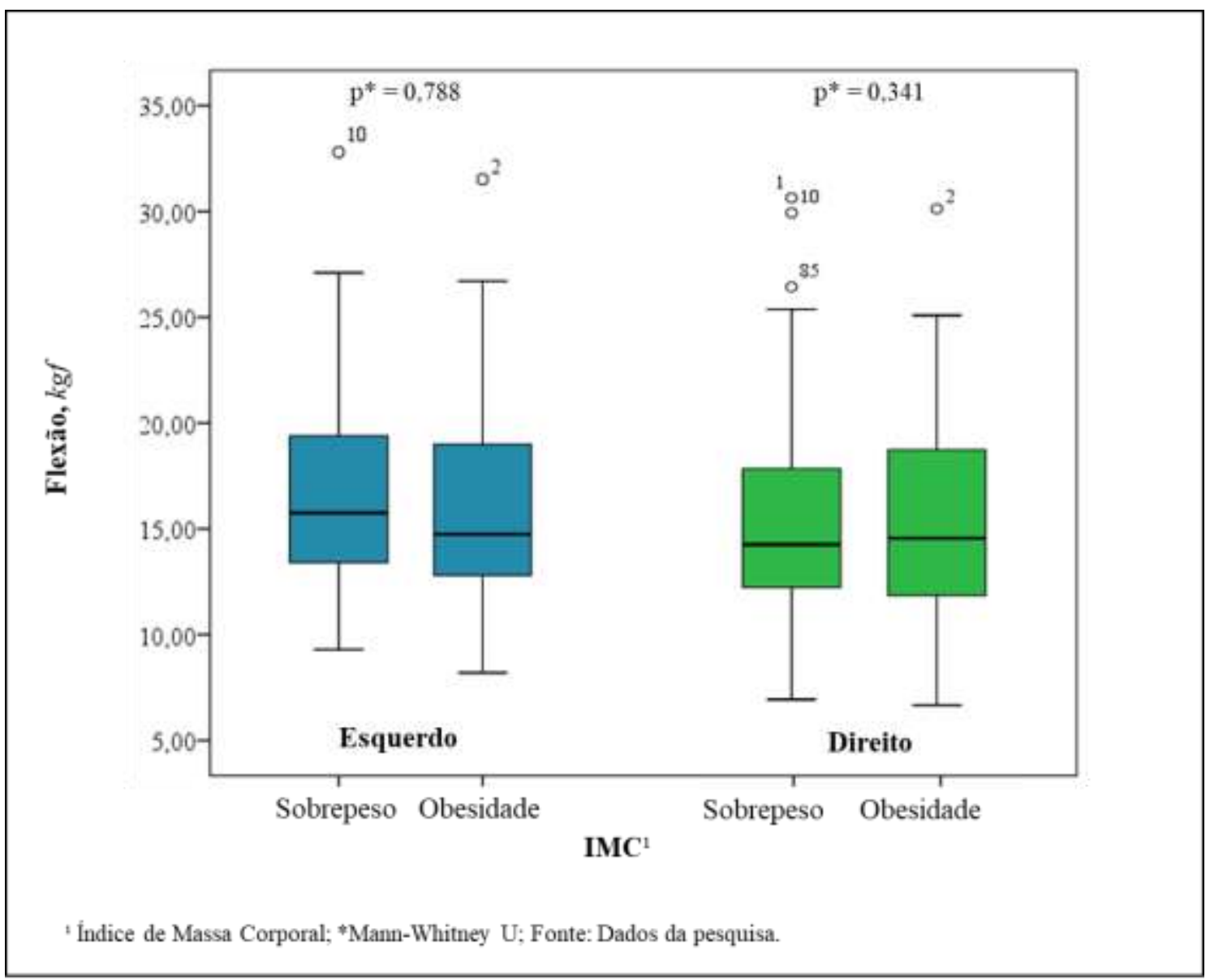

Fonte: Dados da pesquisa.

O mesmo comportamento comparativo entre os grupos foi observado na análise da pelo movimento de extensão. Nos indivíduos sobrepesos, as medidas médias da extensão direita e esquerda foram, 14,11 $( \pm 3,62) \mathrm{kgf}$ e 14,42 ( $\pm 3,66) \mathrm{kgf}$, respectivamente. Enquanto nos indivíduos identificados com obesidade, foram encontrados valores médios da direita e esquerda de em 13,23 ( $\pm 4,19) \mathrm{kgf}$ e 13,28 $( \pm 4,12) \mathrm{kgf}$, nesta ordem. Entretanto, essa diferença entre as médias para os grupos não foi verificada estatisticamente de forma significante em ambas as lateralidades, $(\mathrm{p}=0,288$ e $\mathrm{p}=0,278)$, conforme mostra o Gráfico 4. 
Research, Society and Development, v. 10, n. 16, e503101624216, 2021

(CC BY 4.0) | ISSN 2525-3409 | DOI: http://dx.doi.org/10.33448/rsd-v10i16.24216

Gráfico 4. Distribuição da força dada pela extensão esquerda e direita por IMC. Vitória da Conquista - BA, 2021.

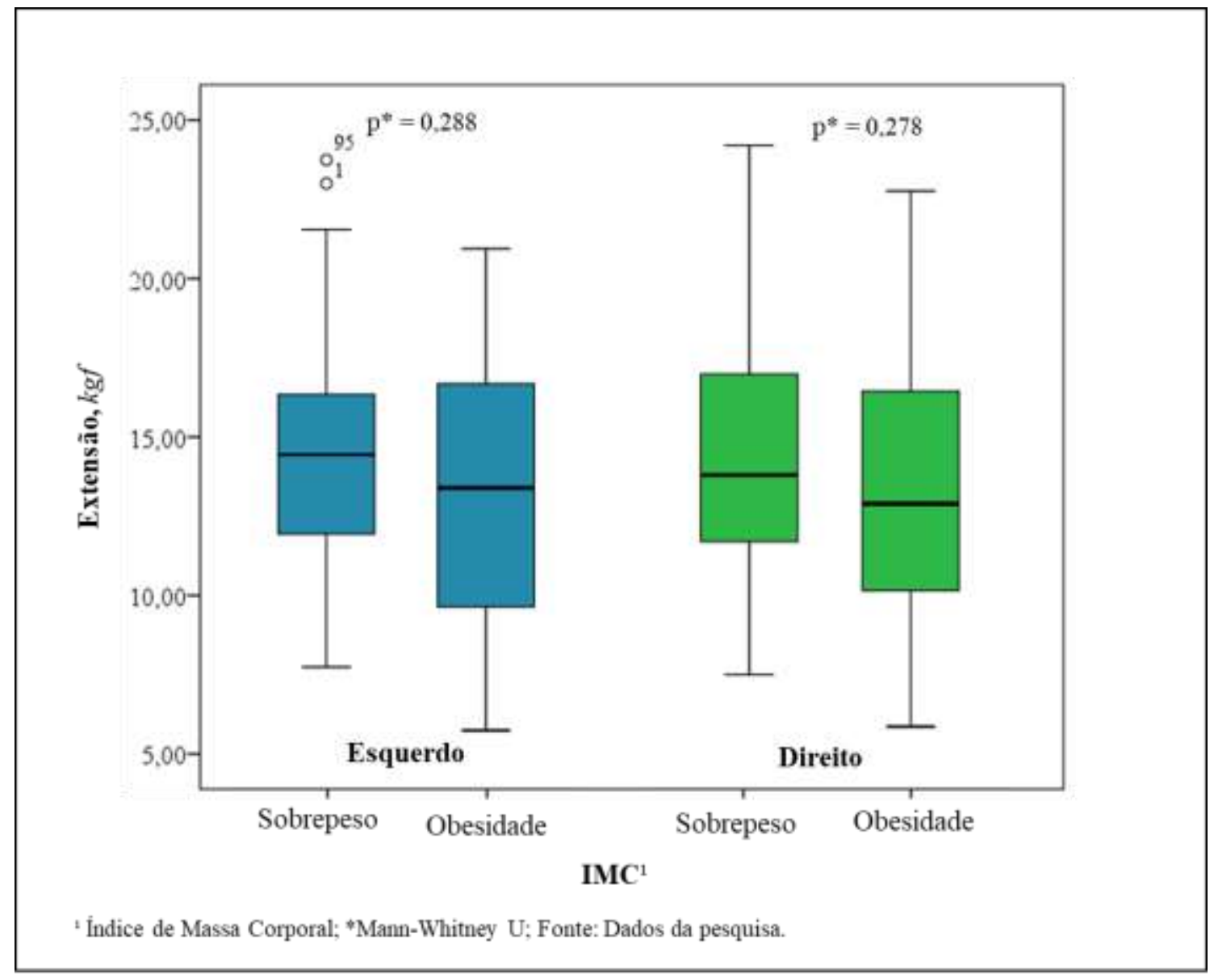

Fonte: Dados da pesquisa.

Tempo de marcha: Ao analisar o Gráfico 5, é possível inferir que não existe diferença significante entre o tempo de marcha entre os grupos $(\mathrm{p}=0,165)$, onde sobrepesos apresentaram média de tempo centrado em $6,77( \pm 1,28) \mathrm{m} / \mathrm{s}$, sendo superior à média dos obesos 7,18 $( \pm 1,38) \mathrm{m} / \mathrm{s}$. 
Gráfico 5. Distribuição do tempo de marcha por IMC da amostra. Vitória da Conquista - BA, 2021.

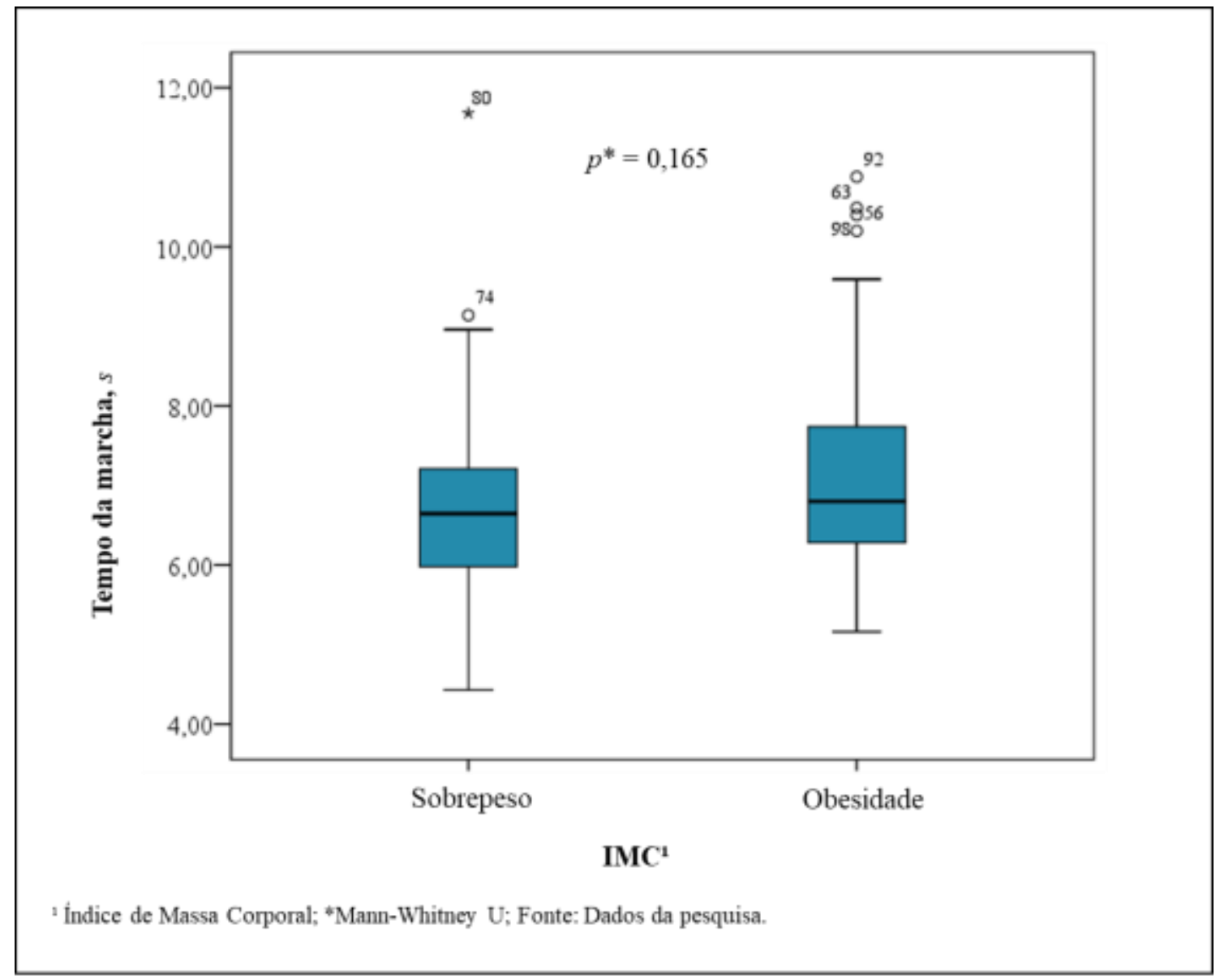

Fonte: Dados da pesquisa.

Diante disto, valores do tempo de marcha inferiores a 8s, foram identificados como possível indicador de sarcopenia na amostra. De acordo com os dados contidos na Tabela 2, observa-se presença de sarcopenia prevalentemente em indivíduos sobrepesos, embora não sejam verificados de forma significante $(\mathrm{p}=0,398)$.

Tabela 2. Características clínicas da amostra. Vitória da Conquista - BA, 2021.

\begin{tabular}{|c|c|c|c|}
\hline \multirow{2}{*}{ Variáveis } & \multicolumn{2}{|c|}{ Grupos, $n(\%)$} & \multirow{2}{*}{$p^{*}$} \\
\hline & Sobrepesos $(n=50)$ & $\operatorname{Obesos}(n=50)$ & \\
\hline \multicolumn{4}{|l|}{ Sarcopenia } \\
\hline Presença & $42(51,2 \%)$ & $40(48,8 \%)$ & \multirow{2}{*}{0,398} \\
\hline Ausência & $8(44,4 \%)$ & $10(55,6 \%)$ & \\
\hline
\end{tabular}

*Teste do qui-quadrado de Pearson; Fonte: Dados da pesquisa.

\section{Discussão}

Os resultados dessa pesquisa evidenciaram uma amostra em indivíduos adultos residentes em uma cidade do interior da Bahia uma prevalência de 50 pessoas obesas com sarcopenia e 47 sobrepesos, tendo assim apenas 3 sem a presença da comorbidade. Esses dados foram culminados de acordo com a análise de um teste de marcha e avaliação da força muscular dos mesmos. 
A Organização Mundial de Saúde (OMS) considera a circunferência da panturrilha (CP) uma medida sensível da massa muscular nos indivíduos, a qual indica alterações que ocorrem com o envelhecimento e com a redução de atividade física. Além disso, já foi até evidenciada associação entre CP inferior a 31 centímetros e incapacidade, assim como, associação elevada de CP com menor nível de fragilidade e melhor desempenho funcional. (Barillaro et al. 2013).

Pode se depreender a caracterização dos indivíduos participantes deste estudo que tem prevalência dos valores de CP acima do limite da referência $(31 \mathrm{~cm})$. Segundo Pastore et al., por conseguinte, a medida pode ter sido influenciada pelo excesso de gordura subcutânea, considerando que já se demonstrou correlação positiva da CP com IMC.

Existem poucos estudos voltados para a avaliação antropométrica em relação a sarcopenia, por isso o método torna-se questionável a erros. Sabendo da não recomendação clínica sobre a medida antropométrica para a identificação da sarcopenia, os desfechos deste estudo mostraram que a CP contribuiu de forma somatória para a ligação dos indivíduos com a comorbidade citada, associada aos outros meios de avaliação utilizados (Cruz-Jentoft et al. 2010).

Com relação aos testes funcionais, utilizamos a caminhada de 4 metros, na qual o indivíduo não poderia ultrapassar a média de $0,8 \mathrm{~m} / \mathrm{s}$, sendo considerável para sarcopenia. Segundo Diz et al. (2015), por ser considerada uma tarefa motora complexa, a marcha depende de diversas capacidades físicas, pois requer energia, controle motor, força, potência muscular e aptidão cardiorrespiratória. Visto as análises, foi deferido que a média dos sobrepesos é maior que a dos obesos, sendo respectivamente 0,60 e 0,57. Contudo, 47 pessoas do primeiro grupo denotaram a comorbidade restando 3 e do grupo dos obesos, todos os 50 indivíduos avaliados apresentaram sarcopenia.

Em outro estudo realizado na qual utilizaram o Time Up and Go (TUG) como teste de marcha, foi observado que a associação de baixa massa muscular e de doenças crônicas contribuíram para maior comprometimento do desempenho físico quando comparado aos fatores isolados. Quanto ao pior desempenho no TUG dos indivíduos avaliados, provavelmente ocorreu devido à diminuição de força e potência de membros inferiores, como também observado por outros autores (Moreira, Perez \& Lourenço, 2019).

Foi observado que independente do teste de marcha realizado, este deve ser levado em consideração para a avaliação da sarcopenia. A ativação e execução cronometrada examina o tempo necessário para realizar uma série de tarefas funcionalmente críticas; também pode servir como uma medida de desempenho (Choi, 2016).

De acordo com Abreu \& Caldas (2008), a velocidade da marcha declina com a idade, entretanto, há uma relação não linear entre a força dos membros inferiores e velocidade da marcha normal. Essa relação representa o mecanismo pelo qual pequenas alterações na capacidade fisiológica podem produzir efeitos substanciais no desempenho demonstrado em adultos frágeis, enquanto que grandes mudanças em adultos saudáveis têm pouco ou nenhum efeito sobre a função diária.

Quanto a força muscular, avaliada através do dinamômetro digital, trouxemos uma correlação com a força de preensão manual, utilizada na grande maioria dos estudos sobre sarcopenia. Esta é uma variável que traduz a força muscular dos membros superiores. O teste é realizado com o indivíduo posicionado sentado, cotovelo fletido a $90^{\circ}$ e antebraço e punho em posição neutra. O método mais utilizado para cálculo dos valores é a média de três tentativas para cada mão (Oliveira, Santos \& Reis, 2017).

No nosso estudo, realizamos o teste de força também com o dinamômetro manual, porém avaliando a força dos indivíduos nos membros inferiores. Foi solicitado que o esse se sentasse com coluna ereta, quadril e joelhos fletidos a $90^{\circ} \mathrm{com}$ o aparelho posicionado na região supra patelar na inserção distal do músculo quadríceps, realizando flexão de quadril por 3 vezes em cada lado; Em seguida deitado em decúbito ventral com joelhos fletidos em $90^{\circ}$ e o aparelho posicionado na região posterior distal do fêmur, realizando extensão de quadril também por 3 vezes. 
O que podemos trazer em associação com esses dois métodos de avaliação para a força, é que ambos são positivos para o diagnóstico de sarcopenia. Contudo, é indicado que mais pesquisas sejam feitas na área e com a avaliação da força dos membros inferiores, visto que não foram encontrados estudos relevantes e confiáveis para a comparação.

A classificação de sarcopenia tem sido realizada de maneira diversificada na literatura e ainda há dúvidas sobre os critérios para sua definição, indicando a necessidade de mais investigações bem controladas para padronizar os critérios diagnósticos dessa síndrome (Paula, 2016).

Quanto maior o peso, menor o resultado satisfatório da bioimpedância, pois ele se refere em menor massa muscular e maior percentual de gordura; o sistema musculoesquelético sofre ao longo dos anos uma diminuição da massa muscular que se inicia a partir da terceira década de vida e é acentuado no começo da quinta. Estima-se uma queda de 1,2\% da massa muscular e 1,3\% da força para cada ano subsequente. Com achados similares o presente estudo confirma que, quanto maior a classificação do IMC, maior a porcentagem de gordura, o que pode acarretar uma fraqueza da musculatura (Lanari, 2012).

Existem algumas limitações na identificação e classificação da sarcopenia. A BIA apesar de ser um método validado, não é considerada "padrão ouro" para avaliar a massa muscular (Paula, 2016).

\section{Conclusão}

Mesmo com as limitações da pesquisa, entre elas a escassez de estudos voltados para a área, foi possível atingir os objetivos e trazer resultados concretos que, de fato, indivíduos jovens com padrão de sobrepeso ou obesidade podem e apresentam sarcopenia.

De acordo com o que foi visto no decorrer da pesquisa, observamos a importância de buscar e manter hábitos de vida saudáveis, pois a sarcopenia acarreta em perdas importantes na qualidade de vida dos indivíduos, trazendo limitações e prejuízos em relação a funcionalidade.

Com isso, sugere-se a realização de novos estudos e investigações bem controladas futuras, afim de abordar mais evidências científicas e atrair resultados em indivíduos obesos e sobrepesos com sarcopenia.

\section{Referências}

Abreu, S. S. E., \& Caldas, C. P. (2008). Velocidade de marcha, equilíbrio e idade: um estudo correlacional entre idosas praticantes e idosas não praticantes de um programa de exercícios terapêuticos. Brazilian Journal of Physical Therapy, 12(4), 324-330. https://doi.org/10.1590/s1413-35552008000400012

Anastácio, L. R., Ferreira, L. G., Ribeiro, H. S., Diniz, K. G. D., Lima, A. S., Correia, M. I. T. D., \& Vilela, E. G. (2019). Sarcopenia, obesity and sarcopenic obesity in liver transplantation: A body composition prospective study. Arquivos Brasileiros de Cirurgia Digestiva [Brazilian Archives of Digestive Surgery], 32(2), e1434. https://doi.org/10.1590/0102-672020190001e143

Barbosa Filho, V. C., Quadros, T. M. B. de, Souza, E. A. de, Gordia, A. P., \& Campos, W. de. (2010). A utilização do critério da Organização Mundial de Saúde para classificação do estado nutricional em crianças. Motriz: Revista de Educacao Fisica. UNESP. https://doi.org/10.5016/1980-6574.2010v16n4p811

Barillaro, C., Liperoti, R., Martone, A. M., Onder, G., \& Landi, F. (2013). The new metabolic treatments for sarcopenia. Aging Clinical and Experimental Research, 25(2), 119-127. https://doi.org/10.1007/s40520-013-0030-0

Carvalho, E. R. \& Rocha, H. A. L. (2016). Estudos Epidemiológicos. Universidade Federal do Ceará. http://www.epidemio.ufc.br/files/06RReOR.pdf

Choi, K. M. (2016). Sarcopenia and sarcopenic obesity. The Korean Journal of Internal Medicine, 31(6), 1054-1060. https://doi.org/10.3904/kjim.2016.193

Cruz-Jentoft, A. J., Baeyens, J. P., Bauer, J. M., Boirie, Y., Cederholm, T., Landi, F., Martin, F. C., Michel, J.-P., Rolland, Y., Schneider, S. M., Topinková, E., Vandewoude, M., Zamboni, M., \& European Working Group on Sarcopenia in Older People. (2010). Sarcopenia: European consensus on definition and diagnosis: Report of the European Working Group on Sarcopenia in Older People. Age and Ageing, 39(4), 412-423. https://doi.org/10.1093/ageing/afq034

Cruz-Jentoft, A. J., Bahat, G., Bauer, J., Boirie, Y., Bruyère, O., Cederholm, T., Cooper, C., Landi, F., Rolland, Y., Sayer, A. A., Schneider, S. M., Sieber, C. C., Topinkova, E., Vandewoude, M., Visser, M., Zamboni, M., \& Writing Group for the European Working Group on Sarcopenia in Older People 2 (EWGSOP2), and the Extended Group for EWGSOP2. (2019). Sarcopenia: revised European consensus on definition and diagnosis. Age and Ageing, 48(1), 16-31. https://doi.org/10.1093/ageing/afy169

Da Fonseca, N. J. (2020) Aplicativo: testes para avaliar a sarcopenia e prescrição de atividade física. Fisioterapia. Dissertação (Mestrado). Universidade do Vale do Sapucaí 
Research, Society and Development, v. 10, n. 16, e503101624216, 2021

(CC BY 4.0) | ISSN 2525-3409 | DOI: http://dx.doi.org/10.33448/rsd-v10i16.24216

De Campos, G. C., Lopes, C. S., \& Lourenço, R. A. (2018). Obesidade sarcopênica e funcionalidade: Uma revisão da literatura. Revista Hospital Universitário Pedro Ernesto, 16(2). https://doi.org/10.12957/rhupe.2017.37650

Diretrizes Brasieiras de Obesidade. $4^{\circ}$ edição. São Paulo: Abeso, 2016.

Diz, J. B. M., Queiroz, B. Z. de, Tavares, L. B., \& Pereira, L. S. M. (2015). Prevalência de sarcopenia em idosos: resultados de estudos transversais amplos em diferentes países. Revista Brasileira de Geriatria e Gerontologia, 18(3), 665-678. https://doi.org/10.1590/1809-9823.2015.14139

IBGE - Instituto Brasileiro de Geografia e Pesquisa. Censo Demográfico - Vitória da Conquista - BA, 2021. https://www.ibge.gov.br/cidades-eestados/ba/vitoria-da-conquista.html

Lanari, A. F. (2012). Sarcopenia repercusiones mecânicas y funcionales. Associacion Argentina de Traumatologia del Deporte, 9, 23-31. http://www.clinicadeldeporte.com.ar/documentos/2012/23_31_SARCOPENIA_9.pdf

Moreira, V. G., Perez, M., \& Lourenço, R. A. (2019). Prevalence of sarcopenia and its associated factors: the impact of muscle mass, gait speed, and handgrip strength reference values on reported frequencies. Clinics (Sao Paulo, Brazil), 74, e477. https://doi.org/10.6061/clinics/2019/e477

Oliveira, E. N. de, Santos, K. T. dos, \& Reis, L. A. dos. (2017). Força de preensão manual como indicador de funcionalidade em idosos. Revista Pesquisa Em Fisioterapia, 7(3), 384-392. https://doi.org/10.17267/2238-2704rpf.v7i3.1509

Paula, J. A. de, Wamser, E. L., Gomes, A. R. S., Valderramas, S. R., Cardoso Neto, J., \& Schieferdecker, M. E. M. (2016). Análise de métodos para detectar sarcopenia em idosas independentes da comunidade. Revista Brasileira de Geriatria e Gerontologia, 19(2), 235-246. https://doi.org/10.1590/180998232016019.140233

Pícoli, T. da S., Figueiredo, L. L. de, \& Patrizzi, L. J. (2011). Sarcopenia e envelhecimento. Fisioterapia Em Movimento, 24(3), 455-462. https://doi.org/10.1590/s0103-51502011000300010

Resolução n ${ }^{\circ}$ 466, de 12 de dezembro de 2012. Dispõe sobre diretrizes e normas regulamentadoras de pesquisas envolvendo seres humanos. Diário Oficial [da] República Federativa do Brasil, Brasília, DF, 13 jun. 2013.

Rocha, G. A., Diniz, K. G. D., Coelho, M. P. P., Vieira, D. A., Soares, M. M. S., Kakehasi, A. M., Correia, M. I. T. D., Teixeira, R., Queiroz, D. M., M., Bering, T. \& Silva, L. D. (2018). Association between pre-sarcopenia, sarcopenia, and bone mineral density in patients with chronic hepatitis C: Sarcopenia and bone mineral density in hepatitis C. Journal of Cachexia, Sarcopenia and Muscle, 9(2), 255-268. https://doi.org/10.1002/jcsm.12269

Tavares, T. B., Nunes, M. S. \& Santos, M. de O. (2010). Obesidade e qualidade de vida: revisão de literatura. RMMG - Revista Médica de Minas Gerais, Contagem - MG, vol. 20 (3), 359-366. http://rmmg.org/artigo/detalhes/371 